\title{
Complete Cessation of Recurrent Cervical Intraepithelial Neoplasia (CIN) by the Paleolithic Ketogenic Diet: A Case Report
}

\author{
Csaba Tóth, Mária Schimmer, Zsófia Clemens* \\ Paleomedicina Hungary Ltd, Evolutionary Medicine Working Group, Budapest, Hungary \\ *Corresponding author: clemenszsofia@gmail.com
}

\begin{abstract}
Background: Ketogenic diets have repeatedly been suggested for the treatment of cancer. To date, only a few case studies reporting long term benefits associated with such diets have been published. Case report: Here we present a case where recurrent cervical intraepithelial neoplasia (CIN), a premalignant condition of cervical cancer, was successfully treated with the paleolithic ketogenic diet. The patient had a history of high grade CIN in 2011, which was successfully treated with cervical conization and fractional curettage. In October 2015 she was found to have a recurrence of high grade CIN (HSIL: High Grade Squamous Intraepithelial Lesion). The patient was then commenced on the paleolithic ketogenic diet and her repeat Pap (Papanicolaou) smear three months later was found to have reverted to normal. The patient has remained on the paleolithic ketogenic diet for 26 months, her repeat smears have stayed normal, and she is free of symptoms and side effects. Conclusion: We conclude that the diet was effective and safe in this patient. As a major benefit the patient was able to avoid a hysterectomy, which is the standard treatment for recurrent high-grade CIN. We believe that the paleolithic ketogenic diet combines benefits of both paleolithic and classical ketogenic diets, while excluding their disadvantages.
\end{abstract}

Keywords: ketosis, evolutionary medicine, dietary intervention, cancer, metabolic therapy

Cite This Article: Csaba Tóth, Mária Schimmer, and Zsófia Clemens, "Complete Cessation of Recurrent Cervical Intraepithelial Neoplasia (CIN) by the Paleolithic Ketogenic Diet: A Case Report." Journal of Cancer Research and Treatment, vol. 6, no. 1 (2018): 1-5. doi: 10.12691/jcrt-6-1-1.

\section{Introduction}

Ketogenic diets have long been suggested as an alternative approach to cancer management [1]. Support for the ketogenic diet comes mainly from studies conducted on animals as well as research at the cellular level [2]. In contrast to the prevailing view of cancer as a predominantly genetic disease, Seyfried [2] suggested cancer as a metabolic disease emerging from respiratory insufficiency coupled with compensatory fermentation, an idea which can be traced back to the original theory of Warburg [3]. Seyfried suggested this altered energy metabolism to be common to all cancer types irrespective of the tissue type [2]. He also suggested that targeting this common metabolic component with the ketogenic diet would be more effective than targeting secondary and more variable components which is the case with many chemotherapy drugs [4].

Although human studies have shown that the classical ketogenic diet is relatively safe [1,5], they have provided little evidence that it is indeed beneficial in cancer. As far as we know only two cases of following the classical ketogenic diet have resulted in long-term survival [6]. Group studies that were carried out with the classical ketogenic diet were either limited in follow up duration [1,7] or reported no or uncertain benefit in terms of survival $[8,9]$.
We have recently published a case where using the paleolithic ketogenic diet without any other treatment resulted in halted progression of soft palate cancer for 20 months [10]. The paleolithic ketogenic diet differs from the classical ketogenic diet in that it excludes food components that were not available in preagricultural times (milk, dairy, cereal grains, oilseeds, nightshades, vegetable oils, food additives, dietary supplements). It has been suggested that non-paleolithic food components induce pathological permeability of the intestine [11] and that of other barrier membranes, a factor thought to be involved in the cancerous transformation of normal tissues [12]. The paleolithic ketogenic diet that we use is close to the animal meat-fat based diet proposed by Voegtlin as being evolutionary adapted for humans [13].

Here we report a case of a patient with recurrent high grade cervical intraepithelial neoplasia (CIN) where following the paleolithic ketogenic diet reversed this condition as evidenced by gynecological cytology.

\section{Results}

\subsection{Previous Medical History}

The patient was a life-long non-smoker, had never used oral contraceptives and had three normal vaginal deliveries. 
In 2011, at the age of 42, a high-grade squamous intraepithelial lesion was found on a routine Pap (Papanicolaou) smear and associated abnormal findings on colposcopy. The patient underwent cervical conization and fractional curettage in June 2011. Excision occurred with free margins. Histopathology of the excized specimen indicated high-grade CIN (HSIL: high-grade squamous intraepithelial lesion).

Between 2012 and 2014 consecutive gynecological ultrasound exams showed that the endometrial thickness decreased from $9.8 \mathrm{~mm}$ (in August 2012) to $2.7 \mathrm{~mm}$ (in January 2014). Testing for human papillomavirus (HPV) DNA, carried out in June 2013, was normal.

In February 2014 the patient started a popular version of the paleolithic diet with the aim of preserving health. The next two ultrasounds showed increasing thickness of the endometrium with $5.2 \mathrm{~mm}$ in December 2014 and 12.5 $\mathrm{mm}$ in May 2015 (Table 1). Due to the thickened endometrium fractional curettage was performed in June 2015. Histopathology indicated high-grade CIN (HSIL).

Pap smears between Sep 2011 and May 2015 were normal, but the smear in October 2015 (20 months after the onset of the popular paleolithic diet and four months after performing the fractional curettage) was found to be abnormal.

HPV DNA testing, performed in August 2015 (after 18 months on the popular paleolithic diet) showed the presence of low-risk types. Based on the abnormal histopathology, the abnormal cytology and the HPV positivity, the patient was offered abdominal hysterectomy. The patient asked for surgery to be postponed and contacted us for dietary guidance with the aim of avoiding a hysterectomy.

\subsection{The Popular Paleolithic Diet}

The patient had been following a popular version of the paleolithic diet for 20 months between February 2014 and October 2015. In her diet vegetables, fruits and oilseeds predominated over animal-based foods. Besides animal fats, her diet also contained vegetable oils including coconut oil and olive oil. She was consuming sugar alcohols as sweeteners and was also taking multiple dietary supplements. She did not consume offal on a regular basis. This version of the paleolithic diet conforms neither to the paleolithic diet as originally described by Cordain [11] or Lindeberg [14] nor any of those versions in previously published clinical studies of the paleolithic diet $[15,16,17,18]$. We therefore refer to this diet as a 'popular paleolithic diet' because it appears to arise from a mixture of scientific and unscientific information, much of which is probably gleaned from dubious sources on the internet.

\subsection{Paleolithic Ketogenic Diet}

The patient was reluctant to accept a hysterectomy that was offered after the diagnosis of recurrent high-grade CIN. Shortly afterwards she contacted us, with the primary goal of avoiding a hysterectomy. We suggested the paleolithic ketogenic diet, which the patient started immediately. The paleolithic ketogenic diet is an animal fat-meat based diet with a fat: protein ratio of about 2: 1 (in grams). The diet excludes cereal grains, milk, other dairy foods, nightshades, oilseeds, vegetable oils (including coconut oil and olive oil), legumes, refined sugars, artificial sweeteners, food additives and all types of dietary supplements. Vegetables and fruits were restricted to less than $30 \%$ in volume. Red and fatty meats and a regular intake of offal from cattle and pork was encouraged. The patient was allowed to consume honey in moderation. Coffee consumption was limited to one coffee a day. The patient has now been on the paleolithic ketogenic diet for 26 months, and is currently still on it.

Table 1. Pap smear, endometrial thickness (as shown by gynecological ultrasound) and HPV DNA testing data on the normal diet, popular paleolithic diet and the paleolithic ketogenic diet. ASC-US: atypical squamous cells of unknown significance

\begin{tabular}{|c|c|c|c|c|c|c|}
\hline & Pap smear & date & Endometrial thickness (mm) & date & HPV DNA & date \\
\hline \multirow{5}{*}{ Normal diet } & abnormal & 24.05 .2011 & & $\cdot$ & & \\
\hline & \multicolumn{6}{|c|}{ Conization, fractional curettage 21.06 .2011} \\
\hline & normal & 27.09 .2011 & 9.8 & 29.08 .2012 & & \\
\hline & normal & 15.10 .2013 & 8.4 & 15.10 .2012 & negative & 07.07 .2013 \\
\hline & normal & 28.01 .2014 & 2.7 & 29.01 .2014 & & \\
\hline \multirow{6}{*}{ Popular Paleolithic diet } & normal & 08.04 .2014 & & & & \\
\hline & normal & 16.09.2014 & & & & \\
\hline & normal & 20.01 .2015 & 5.2 & 12.12 .2014 & & \\
\hline & normal & 19.05 .2015 & 12.5 & 21.05 .2015 & & \\
\hline & \multicolumn{6}{|c|}{ Fractional curettage 09.06 .2015} \\
\hline & abnormal & 08.10 .2015 & & & positive & 28.08 .2015 \\
\hline \multirow{6}{*}{ Paleolithic ketogenic diet } & normal & 14.01 .2016 & & & & \\
\hline & normal & 28.04 .2016 & & & & \\
\hline & ASC-US & 30.09 .2016 & & & & \\
\hline & normal & 25.01 .2017 & & & & \\
\hline & normal & 09.08 .2017 & & & & \\
\hline & normal & 01.12 .2017 & & & & \\
\hline
\end{tabular}




\subsection{Follow up}

\subsubsection{Laboratory Workup and Urinary Ketones}

A laboratory workup after 11 months on a paleolithic ketogenic diet (September 2016) was normal, and the low triglyceride and uric acid levels and mildly elevated cholesterol level were compatible with following the paleolithic ketogenic diet. Inflammation level as indicated by CRP was also low. The low thyroid stimulating hormone (TSH) level of the patient is compatible with a low carbohydrate diet (Table 2). The patient used urinary ketone strips on a daily basis which showed sustained ketosis except for during the summer months of 2016 when she reported 15 days of not being in ketosis and which was likely due to an increased consumption of fruits.

Table 2. Laboratory measurements on the paleolithic ketogenic diet (on 20 Sep 2016)

\begin{tabular}{lccc}
\hline & & reference range & $\mathrm{unit}$ \\
\cline { 2 - 4 } CRP & 0.66 & $0-10$ & $\mathrm{mg} / 1$ \\
Glucose & 5.8 & $3.6-6.1$ & $\mathrm{mmol} / 1$ \\
Carbamide & 4 & $2.5-7.5$ & $\mathrm{mmol} / 1$ \\
Creatinine & 55 & $40-88$ & $\mu \mathrm{mol} / 1$ \\
Total cholesterol & 6.6 & $2.8-5.2$ & $\mathrm{mmol} / 1$ \\
HDL cholesterol & 1.87 & $1.09-2.29$ & $\mathrm{mmol} / 1$ \\
Triglyceride & 0.7 & $0.9-1.87$ & $\mathrm{mmol} / 1$ \\
Uric acid & 222 & $178-345$ & $\mu \mathrm{mol} / 1$ \\
GOT & 16 & $0-37$ & $\mathrm{U} / 1$ \\
GPT & 11 & $0-40$ & $\mathrm{U} / 1$ \\
GGT & 13 & $7-32$ & $\mathrm{U} / 1$ \\
Iron & 11.8 & $10.7-32.2$ & $\mu \mathrm{mol} / 1$ \\
WBC & 4.39 & $4.5-11$ & $\mathrm{G} / 1$ \\
RBC & 4.09 & $4-5.2$ & $\mathrm{~T} / 1$ \\
Hemoglobin & 12.8 & $12-15.5$ & $\mathrm{~g} / \mathrm{dl}$ \\
Hematocrit & 37.2 & $36-50$ & $\%$ \\
Thrombocyte & 229 & $150-350$ & $\mathrm{G} / 1$ \\
TSH & 0.74 & $0.550-4.780$ & $\mu \mathrm{IU} / \mathrm{ml}$ \\
\hline
\end{tabular}

Abbreviations:

CRP: C-reactive protein, HDL: high density lipoprotein,

GOT: glutamate-oxaloacetate transaminase, GPT: glutamate-pyruvate transaminase, GGT: gamma-glutamyl transferase, WBC: white blood cell count, RBC: red blood cell count, TSH: thyroid stimulating hormone.

\subsubsection{Cervical Cytology}

During the follow up of 26 months the patient had six cervical cytology tests (Table 1). The first cytology follow up, performed at three months after diet onset (in January 2016), as well as the second one at six months on the diet (in April 2016) were normal. The third follow up at one year after diet onset (in Sep 2016) indicated atypical squamous cells of unknown significance (ASC-US) but no neoplastic alteration. This third cervical cytology was performed after a period of poor dietary compliance during which the patient was not in ketosis. After learning about the result of the abnormal cytology the patient went back to following the diet strictly. The fourth follow up, which was performed in January 2017 after going back to being compliant with the diet, showed normal results again. The next two follow ups (in August 2017 and December 2017) also showed normal results.

\subsubsection{General Condition}

At diet onset the patient weighed $55 \mathrm{~kg}(\mathrm{BMI}=20.7)$. On the paleolithic ketogenic diet she lost two kilograms. Currently her BMI is 19.9. She reports feeling generally well, and has no symptoms or side-effects due to the diet.

\section{Discussion}

CIN is regarded as a premalignant condition of cervical cancer [19]. Recurrence of cervical intraepithelial lesion after treatment is usually regarded as an indication for hysterectomy, a radical treatment of CIN. Upon recurrence our patient was offered a hysterectomy too. However, she opted for delaying hysterectomy and started the paleolithic ketogenic diet. Importantly, for the four years after the first conization and the fractional curettage she was disease-free as evidenced by regular gynecological cytology and ultrasound. Yet, while on the popular paleolithic diet, ultrasound showed endometrial thickening and both HPV DNA test and cervical cytology turned positive.

The diet denoted as "paleolithic" in the medical literature has been shown to confer several benefits in metabolic syndrome and associated conditions as also evidenced by a metaanalysis [20]. By definition, the paleolithic diet excludes foods that were not available in prehistoric times $[11,14]$. However, the paleolithic diet as published in clinical studies $[15,16,17,18]$ differ from the diet we refer to as paleolithic ketogenic in important aspects including macronutrient ratios and the ratio of animal vs. plant based food components. Moreover, given the availability of internet-based, mainly non-scientific resources, several variants of the paleolithic diet abound which we collectively denote as popular paleolithic diet. These diets may also contain food components that should not be denoted as "paleolithic" according to Cordain [21].

We believe that the paleolithic diet (as described in previously published clinical studies) is inappropriate to fight autoimmune diseases and cancer. As we previously reported, recovery from Crohn's disease using the paleolithic ketogenic diet may be hindered by eating components of the popular paleolithic diet [22]. In another case study we reported that the popular paleolithic diet did not confer all the benefits that were provided by the paleolithic ketogenic diet in Gilbert's syndrome [23]. Previously we have published a case of soft palate cancer halted by the paleolithic ketogenic diet for 20 months [10]. We also reported successful management of cases of autoimmune conditions including type 1 diabetes [24,25] and cases with epilepsy [26,27] and metabolic syndrome [28] using the paleolithic ketogenic diet. In another case study we reported that a full dietary adherence is needed to halt disease progression in rectal cancer [29]. In a reply paper we summarized why the paleolithic ketogenic diet may be superior to the classical version of the ketogenic diet and why the standard treatment may be counter effective in the treatment of cancer [30].

Our patient with recurrent CIN started the paleolithic ketogenic diet under our guidance. Three months after diet onset her Pap smear cytology was reported as normal. 
Follow up at three and six months after diet onset her Pap smear cytology was reported as normal. However, the 12 months follow up showed atypical cells of uncertain significance. This slightly abnormal Pap smear was preceded by a period of increased fruit intake and consequently the patient not being in ketosis. The patient then went back to following the paleolithic ketogenic diet strictly and her follow up tests at 15, 22 and 26 months were normal again.

Fluctuation of the grade of the cervical squamous cell pathology is common. However, in the long run the overall tendency of $\mathrm{CIN}$ is progressive even after treatment. Therefore a complete normalization of cervical cytology without any other treatment in a patient with recurrent high grade CIN is unlikely to be part of the normal course of the disease.

Ketogenic diets have been shown to be effective in controlling tumor growth in animal models [2]. In humans, the classical ketogenic diet has been shown to confer benefits in terms of reducing chemo/radiotherapy induced side-effects [2] but not in terms of prolonging survival. Previously, we reported a case with aggressive soft palate cancer successfully managed with the paleolithic ketogenic diet alone [10]. We believe that the state of ketosis, although required, is insufficient on it's own in controlling tumor growth in cancer patients.

Increased permeability of the intestines and that of other membranes is known to be associated with an elevated level of inflammatory markers [31]. Both decreased barrier function [12] and inflammation are known to be associated with the development and growth of tumors [32]. We believe that the paleolithic ketogenic diet by excluding "non-paleolithic" food components normalizes the pathological permeability of the intestines as also seen in a published case of Crohn's disease [22], and likely through the normalization of intestinal permeability results in lowering inflammatory factors (as also showed in our previous case reports). In addition, ketosis, based on animal meat and fat may ensure optimal supply and bioavailability of vitamins, including vitamin $C$ [33] the high blood level of which is important for the proper functioning of the immune system and also convey antitumor effects [34].

It is known that within the mitochondria beta oxidation of fatty acids is associated with the production of matrix water which is relatively low in deuterium. Depletion of deuterium has been suggested as one of the key elements of the antitumor effect of high fat diets. As a potential mechanism of action, it has been suggested that hydrogen bonding in DNA would be negatively impacted by elevated deuterium content given that NADPH-dependent DNA synthesis is subserved by the matrix water pool [35]. As a clinical parallel, there are metabolic profiling data showing that in lung cancer patients rapid progression was associated with serum markers of glycogenic substrates while ketogenic substrates were associated with prolonged survival [36]. More specifically, the importance of the quality and source of dietary fat in determining deuterium content and ratios was also stressed [37].

We conclude that the paleolithic ketogenic diet was safe and effective in our patient with recurrent cervical displasia. We believe that the paleolithic ketogenic diet combines benefits of both paleolithic and classical ketogenic diets, while excluding their disadvantages. By using the paleolithic ketogenic diet the patient was able to avoid a hysterectomy which is thought to be otherwise inevitable knowing the progressive nature of this condition. As a major benefit the patient could preserve her cervix, reproductive capacity, social and sexual integrity, all of which contribute to general well-being.

\section{Acknowledgements}

We are very grateful to Madhvi Chanrai for helping in the review of the manuscript.

\section{Funding}

No funding was received.

\section{Conflict of Interest}

The authors declare that they have no conflict of interest.

\section{Informed Consent}

Informed consent was obtained from the patient included in the study.

\section{References}

[1] Schmidt M., Pfetzer N, Schwab M, Strauss I, Kämmerer U. Effects of a ketogenic diet on the quality of life in 16 patients with advanced cancer: A pilot trial. Nutr Metab (Lond) 2011; 8: 54.

[2] Seyfried TN. Cancer as a Metabolic Disease: On the Origin, Management and Prevention of Cancer, John Wiley \& Sons, Inc., Hoboken, NJ, USA 2012.

[3] Warburg O. On the Origin of Cancer Cells. Science, 1956; 123: 309-14.

[4] Seyfried TN, Flores RE, Poff AM, D'Agostino DP. Cancer as a metabolic disease: implications for novel therapeutics. Carcinogenesis 2014; 35: 515-527.

[5] Klement RJ, Sweeney RA. Impact of a ketogenic diet intervention during radiotherapy on body composition: I. Initial clinical experience with six prospectively studied patients. BMC Research Notes 2016; 9: 143.

[6] Nebeling LC, Miraldi F, Shurin SB, Lerner E. Effects of a ketogenic diet on tumor metabolism and nutritional status in pediatric oncology patients: two case reports. J Am Coll Nutr 1995; 14: $202-8$.

[7] Fine EJ, Segal-Isaacson CJ, Feinman RD, Herszkpf S, Romano $\mathrm{MC}$, Tomuta N, et al. Targeting insulin inhibition as a metabolic therapy in advanced cancer: a pilot safety and feasibility dietary trial in 10 patients. Nutrition 2010; 28: 1028-35.

[8] Rieger J, Bähr O, Maurer GD, Hattingen E, Franz K, Brucker D, et al. ERGO: a pilot study of ketogenic diet in recurrent glioblastoma. Int J Oncol 2014; 44: 1843-52

[9] Champ, CE, Palmer, JD, Volek, JS, Werner-Wasik M, Andrews DW, Evans JJ, Glass J, Kim L, Shi W. Targeting metabolism with a ketogenic diet during the treatment of glioblastoma multiforme. J Neurooncol 2014; 117: 125-31.

[10] Tóth C, Clemens Z. Halted progression of soft palate cancer in a patient treated with the paleolithic ketogenic diet alone: A 20-months Follow-up. Am J Med Case Rep 2015; 4: 288-292.

[11] Cordain L. The paleo diet: lose weight and get healthy by eating the food you were designed to eat. New York: Wiley. 2002. 
[12] Lin JE, Snook AE, Li P, Stoecker BA, Kim GW, Magee MS GUCY2C opposes systemic genotoxic tumorigenesis by regulating AKT-dependent intestinal barrier integrity. PLoS ONE 2012; 7: 2, e31686.

[13] Voegtlin WL. The stone age diet: based on in-depth studies of human ecology and the diet of man. New York: Vantage Press 1975.

[14] Lindeberg S. Food and western disease: health and nutrition from an evolutionary perspective. Chichester: West Sussex, UK, WileyBlackwell 2009.

[15] Jönsson T, Granfeldt Y, Ahrén B, Brannel UC, Pålsson G, Söderström M, et al. Beneficial effects of a Paleolithic diet on cardiovascular risk factors in type 2 diabetes: A randomized crossover pilot study. Cardiovase Diabetol 2009; 16, 8: 35 .

[16] Frassetto LA, Schloetter M, Mietus-Synder M, Morris RC Jr, Sebastian A. Metabolic and physiologic improvements from consuming a paleolithic, hunter-gatherer type diet. Eur J Clin Nutr 2009; 63, 947-55.

[17] Mellberg C, Sandberg S, Ryberg M, Erickson M, Brage S, Larsson $\mathrm{C}$, et al. Long-term effects of a Palaeolithic-type diet in obese postmenopausal women: A 2-year randomized trial. Eur J Clin Nutr 2014; 68: 3507.

[18] Boers I., Muskiet FA, Berkelaar E, Schut E, Penders R, Hoenderdos K, et al. Favourable effects of consuming a Palaeolithic-type diet on characteristics of the metabolic syndrome: a randomized controlled pilot-study. Lipids Health Dis 2014; 13: 160 .

[19] Nelson JH, Averette HE, Richart RM. Cervical Intraepithelial Neoplasia (Dysplasia and Carcinoma in Situ) and Early Invasive Cervical Carcinoma. CA Cancer J Clin 1989; 39, 157-178.

[20] Manheimer EW, van Zuuren EJ, Fedorowicz Z, Pijl H. Paleolithic nutrition for metabolic syndrome: systematic review and metaanalysis. Am J Clin Nutr 2015; 102: 922-32.

[21] Cordain C. Alfalfa sprouts and autoimmune disease. $2014 \mathrm{http}$ //thepaleodiet.com/alfalfa-sprouts-autoimmune-disease, accessed: 30 May 2017

[22] Tóth C, Dabóczi A. Howard M, Miller NJ, Clemens Z. Crohn's disease successfully treated with the paleolithic ketogenic diet. Int J Case Rep Images 2016; 7: 570-578.

[23] Tóth C, Clemens Z. Gilbert's syndrome successfully treated with the paleolithic ketogenic diet. Am J Med Case Rep 2015; 3: $117-120$.

[24] Tóth C, Clemens Z. Type 1 diabetes mellitus successfully managed with the paleolithic ketogenic diet. Int J Case Rep Images 2014; 5: 699-703.
[25] Tóth C, Clemens Z. A child with type 1 diabetes mellitus (T1DM) successfully treated with the Paleolithic ketogenic diet: A 19-month insulin freedom. Int J Case Rep Images 2015; 6: 752-757.

[26] Clemens Z, Kelemen A., Fogarasi A, Tóth C. Childhood absence epilepsy successfully treated with the paleolithic ketogenic diet. Neurol Ther 2013; 2: 71-6.

[27] Clemens Z, Kelemen A, Tóth C. NREM-sleep Associated Epileptiform Discharges Disappeared Following a Shift toward the Paleolithic Ketogenic Diet in a Child with Extensive Cortical Malformation. Am J Med Case Rep 2015; 3: 212-215.

[28] Tóth C, Clemens Z. Successful treatment of a patient with obesity, type 2 diabetes and hypertension with the paleolithic ketogenic diet. Int J Case Rep Images 2015; 6: 161-167.

[29] Tóth C, Clemens Z. Treatment of rectal cancer with the paleolithic ketogenic diet: A 24-months follow-up. Am J Med Case Rep 2017 5: 205-216.

[30] Tóth C, Dabóczi A, Chanrai M, Clemens Z. Comment on "Systematic review: isocaloric ketogenic dietary regimes for cancer patients" by Erickson et al. J Cancer Res Treatment. 2017; $5,86-88$.

[31] Odenwald MA, Turner JR. Intestinal permeability defects: is it time to treat? Clin Gastroenterol Hepatol 2013; 11: 1075-83.

[32] Coussens LM., Werb Z. Inflammation and cancer. Nature 2002; 420: 860-867.

[33] Clemens Z, Tóth C. Vitamin C and Disease: Insights from the Evolutionary Perspective, Journal of Evolution and Health 2016; 1 , 1: 13 .

[34] Krone CA, Ely JT. Controlling hyperglycemia as an adjunct to cancer therapy. Integr Cancer Ther 2005; 4: 25-31.

[35] Boros LG., Meuillet EJ, Somlyai I, Jancsó G, Jákli G, Krempels K, et al. Fumarate hydratase and deuterium depletion control oncogenesis via NADPH-dependent reductive synthesis: mitochondrial matrix water, DNA deuteration and epigenetic events. Cancer Research 2014; 74, 19 Supplement: 1426.

[36] Hao D, Sarfaraz MO, Farshidfar F, Bebb DG, Lee CY, Card CM, David M, Weljie AM. Temporal characterization of serum metabolite signatures in lung cancer patients undergoing treatment. Metabolomics. 2016; 12: 58

[37] Boros LG, Collins TQ, Somlyai G. What to eat or what not to eatthat is still the question. Neuro Oncol. 2017; 19: 595-596. 